\title{
Characteristics and outcomes of patients with COVID-19 at a district general hospital in Surrey, UK
}

\author{
Authors: Harry Knights, ${ }^{\mathrm{A}}$ Nikhil Mayor, ${ }^{\mathrm{A}}$ Kristina Millar, ${ }^{\mathrm{A}}$ Matthew Cox, ${ }^{\mathrm{A}}$ Evgeniya Bunova, ${ }^{\mathrm{A}}$ Morgan Hughes, ${ }^{\mathrm{A}}$ \\ Jack Baker, ${ }^{A}$ Sanju Mathew, ${ }^{B}$ David Russell-Jones ${ }^{C}$ and Aleksandra Kotwica ${ }^{A}$
}

\section{Background}

This retrospective cohort study aims to define the clinical findings and outcomes of every patient admitted to a district general hospital in Surrey with COVID-19 in March 2020, providing a snapshot of the first wave of infection in the UK. This study is the first detailed insight into the impact of frailty markers on patient outcomes and provides the infection rate among healthcare workers.

\section{Methods}

Data were obtained from medical records. Outcome measures were level of oxygen therapy, discharge and death. Patients were followed up until 21 April 2020.

Results

108 patients were included. 34 (31\%) died in hospital or were discharged for palliative care. $43 \%$ of patients aged over 65 died. The commonest comorbidities were hypertension $(49 ; 45 \%)$ and diabetes $(25 ; 23 \%)$. Patients who died were older (mean difference \pm SEM, $13.76 \pm 3.12$ years; $p<0.0001$ ) with a higher NEWS2 score (median 6, IQR 2.5-7.5 vs median 2, IQR 2-6) and worse renal function (median differences: urea $2.7 \mathrm{mmol} / \mathrm{L}, \mathrm{p}<0.01$; creatinine $4 \mu \mathrm{mol} / \mathrm{L}, \mathrm{p}<0.05$; eGFR $14 \mathrm{~mL} / \mathrm{min}, \mathrm{p}<0.05$ ) on admission compared with survivors. Frailty markers were identified as risk factors for death. Clinical Frailty Scale (CFS) was higher in patients over 65 who died than in survivors (median 5, IQR 4-6 vs 3.5, IQR 2-5; $p<0.01$ ). Troponin and creatine kinase levels were higher in patients who died than in those who recovered $(p<0.0001)$. Lymphopenia was common (median 0.8, IQR 0.6-1.2; $\mathrm{p}<0.005$ ). Every patient with heart failure died (8). 26 (24\%) were treated with continuous positive airway pressure (CPAP; median 3 days, IQR 2-7.3) and 9 (8\%) were intubated (median 14 days, IQR 7-21). All patients who died after discharge $(4 ; 6 \%)$ were care home residents. 276 of 699 hospital staff tested were positive for COVID-19.

Authors: Ajunior doctor, Royal Surrey County Hospital, Guildford, UK; ${ }^{B}$ Consultant gastroenterologist, Royal Surrey County Hospital, Guildford, UK; ' Cprofessor of diabetes and endocrinology, Royal Surrey County Hospital, Guildford, UK and University of Surrey, Guildford, UK. Harry Knights and Nikhil Mayor contributed equally to this work

\section{Conclusions}

This study identifies older patients with frailty as being particularly vulnerable and reinforces government policy to protect this group at all costs.

KEYWORDS: Coronavirus, SARS-CoV-2, frailty, COVID-19, Clinical Frailty Scale

DOI: $10.7861 /$ clinmed.2020-0303

\section{Introduction}

Information about the epidemiological and clinical features of adult patients with coronavirus disease 2019 (COVID-19) comes largely from Chinese, ${ }^{1}$ Italian $^{2}$ and American ${ }^{3}$ studies. However, information about this disease and its characteristics in the UK is now emerging. ${ }^{4}$

Initially reported in south-east China in November 2019 and declared a pandemic on 11 March 2020, COVID-19 has spread rapidly across the globe. The first plausible community transmission in the UK was detected on 29 February in Haslemere in Surrey, ${ }^{5}$ south England. This is within the catchment area of the Royal Surrey NHS Foundation Trust / Royal Surrey County Hospital, a district general hospital serving a population of 320,000 for emergency and general hospital services with approximately 3,500 staff. 6

This study describes the population characteristics and outcomes of every patient with COVID-19 admitted to the hospital in March 2020. It includes a comprehensive evaluation of all patients who recovered or died, identifying demographic, clinical and laboratory predictors of poor outcome. All patient outcomes, including those in patients discharged to care homes, were collected. Confirmed healthcare worker infection rate is reported. This study is an early and complete description of patients admitted to hospital with COVID-19 in the UK and provides a snapshot of the first wave of the infection in this country.

Frailty is widely recognised as a risk factor for poor outcome in patients admitted to hospital, with various frailty markers validated as predictors of prognosis in non-COVID-19 patients. ${ }^{7}$ However, frailty is yet to be considered in the context of the COVID-19 pandemic. Here we assess, in detail, frailty markers and their impact on COVID-19 outcomes for the first time. 


\section{Methods}

\section{Data collection}

Data from all patients admitted to the Royal Surrey County Hospital with a positive COVID-19 test from 1-31 March 2020 were collected. All patients were followed until the study endpoint on 21 April 2020.

Epidemiological, clinical, laboratory and radiological characteristics, as well as treatment and outcome data, were obtained. Data were collected from a combination of electronic and paper medical records, as well as from patients directly, and stored using a standardised and secure electronic database. The National Early Warning Score 2 (NEWS2) score was used to stratify patients at risk of deterioration at the time of admission. The frailty markers assessed included polypharmacy, cognitive impairment, dementia, falls, mobility aids, package of care, and care home residence. The Clinical Frailty Scale (CFS) was assessed in all patients above age 65 , as per national guidelines. ${ }^{8}$

All patients underwent venous thromboembolism (VTE) assessment and all received VTE prophylaxis as per local guidelines unless they were anticoagulated prior to admission. As per local microbiology guidelines created in response to COVID-19, all patients received prophylactic antibiotics on admission. All patients had a plain chest radiograph within 24 hours of admission, reported using the British Institute of Radiology COVID-19 Guidelines and Reporting Templates, March 2020.9

Maximum levels of oxygen therapy were categorised as low $(<4 \mathrm{~L} / \mathrm{min})$, high $(>4 \mathrm{~L} / \mathrm{min})$, continuous positive airway pressure (CPAP) and invasive ventilation. Maximum $\mathrm{FiO}_{2}$ was determined for patients receiving CPAP and invasive ventilation. Full escalation was defined as admission to the intensive care unit for intubation and invasive ventilation.

A pragmatic and holistic approach was taken towards ceiling of care decisions. Each patient had their escalation status established at presentation, involving the patient, their family, the admitting consultant physician and intensive care consultant. Patients were discharged based on the clinical judgement of senior clinicians. Criteria for discharge included having no oxygen requirement and the availability of appropriate discharge care. Outcome measures included recovery and discharge, ongoing hospital treatment, and death. The in-hospital deaths included patients discharged for palliative care either at home or a local palliative care inpatient unit. Non-palliative patients who died after discharge are included as survivors in the analysis.

\section{Ethics}

The study was approved by the local Patient Safety and Quality Control Committee. The Medical Research Council ethics decision tool indicates that this research does not require review by an NHS Research Ethics Committee in England.

\section{Informed consent}

As all patient data were anonymised, informed consent was not deemed necessary for this study, in line with guidance from the local Patient Safety and Quality Control Committee.

\section{Laboratory measurements}

Nasopharyngeal, oropharyngeal or bronchoalveolar lavage samples were collected from patients for the extraction of
SARS-CoV-2 RNA. To extract total RNA, commercial isolation kits (ELITe InGenius ${ }^{\mathrm{TM}}$ and Thermofisher Flex MagMAX) were used. The genesig ${ }^{\circledR}$ (Primerdesign ${ }^{T M}$ Ltd) real-time RT-PCR assay was performed to achieve qualitative detection of SARS-CoV-2 RNA. Laboratory investigations included full blood count, renal biochemistry and liver enzyme tests. COVID-19 blood panel tests including lactate dehydrogenase (LDH), creatine kinase (CK), ferritin, d-dimer, high-sensitivity troponin I and C-reactive protein (CRP) were run using routine validated automated clinical assays. For all assays, Siemens machines were used $\left(\right.$ ADVIA $^{\circledR}$ Centaur XP, ADVIA ${ }^{\circledR} 1800$, ADVIA $^{\circledR} 2120 i$ ) with the exception of $d$-dimer (Stago STA-R Evolution ${ }^{\circledR}$ ). Only blood tests performed within 24 hours of admission were included.

\section{Statistical analysis}

Categorical variables are presented as numbers (percentages). Continuous variables are presented as means ( \pm SEM) if normally distributed, or median (interquartile range, IQR) if not. Means for continuous variables were compared using an unpaired t-test. Medians for non-normally distributed data were compared using a Mann-Whitney $U$ test. Categorical variables were compared using the $\chi^{2}$ test. Fisher's exact test was used in the analysis of contingency tables when sample sizes were small. For unadjusted comparisons, a two-tailed p-value below 0.05 was considered to be statistically significant. Statistical analysis was performed using GraphPad Prism v8.4.2.

\section{Results}

\section{Demographics and clinical characteristics}

From 1-31 March 2020, 108 patients were admitted to the hospital with confirmed COVID-19 (age 68.7 \pm 1.5 years; $42 \%$ female; $76 \%$ white British). 65 (60\%) patients were aged over 65 . The most frequent comorbidities were hypertension ( $49 ; 45 \%)$, obesity $(31 / 100 ; 31 \%)$ and diabetes $(25 ; 23 \%)$ (supplementary material S1). 44/101 (44\%) patients were ex- or current smokers and $57 / 101$ (56\%) were never smokers. Frailty was common in this cohort (Table 1). 49 (50\%) patients had at least one frailty marker. $48(44 \%)$ patients were taking five or more medications (defined as polypharmacy). 29/98 (30\%) patients required a form of mobility aid (stick, frame or wheelchair) prior to admission. 11 (10\%) were care home residents and 20/94 (21\%) had a package of care in place. A high proportion of patients $(21 ; 19.4 \%)$ had known cognitive impairment. The median CFS in those aged over 65 was 5 (IQR 4-6).

The median duration of symptoms prior to admission was 7 (IQR $2.3-8.8)$ days. The most common presenting complaints included fever $(84 ; 78 \%)$, cough $(82 ; 76 \%)$ and dyspnoea $(68 ; 63 \%)$. A sizeable proportion of patients presented with gastrointestinal symptoms (24;22\%) such as nausea, vomiting or diarrhoea. Delirium (23; $21 \%)$ and falls $(9 ; 8 \%)$ were also common presenting complaints (Fig 1a).

At triage, 74 (69\%) patients had oxygen saturations $\leq 92 \%$. $68(63 \%)$ had a respiratory rate $>20$, and $50(46 \%)$ had a temperature $\geq 37.8^{\circ} \mathrm{C} .21$ (19\%) presented with a NEWS2 score of greater than 7 (Fig 1b), indicating the need for an urgent or emergency response. The proportion of patients with findings on admission suggestive of COVID-19 on chest radiographs was $66(61 \%)$. 
Table 1. Admission frailty markers of deceased and recovered patients. Values are numbers (percentages)

\begin{tabular}{|c|c|c|c|c|}
\hline Frailty markers & Total $(n=108)$ & Survivors $(n=69)$ & Deaths $(n=34)$ & p-value \\
\hline Polypharmacy* & $48(44)$ & $25(36)$ & $23(68)$ & 0.0027 \\
\hline Cognitive impairment* & $21(19)$ & $10(14)$ & $11(32)$ & 0.034 \\
\hline Dementia & $16(15)$ & $7(10)$ & $9(26)$ & 0.0315 \\
\hline Falls & $19(18)$ & $6(9)$ & $13(38)$ & 0.0003 \\
\hline Mobility aids & $29 / 98(30)$ & $13 / 64(20)$ & $16 / 29(55)$ & 0.0008 \\
\hline Package of care & $20 / 94(21)$ & 10/61 (16) & $10 / 28(36)$ & 0.0426 \\
\hline Care home resident & $11(10)$ & $7(10)$ & $4(12)$ & 0.80 \\
\hline
\end{tabular}

Five patients remained inpatients at the study endpoint and are not included in survivors or deaths. *Polypharmacy $\geq 5$ medications. Cognitive impairment includes formally diagnosed dementia.

\section{Admission laboratory parameters}

Lymphocyte count was low in this cohort (median 0.8, IQR 0.6-1.2; $\mathrm{p}<0.005$ ). Poorer renal function was observed in non-survivors, who had higher urea and creatinine levels (median difference $2.7 \mathrm{mmol} / \mathrm{L}, \mathrm{p}<0.01$ and $4 \mu \mathrm{mol} / \mathrm{L}, \mathrm{p}<0.05$ ) and lower eGFR (median difference $14 \mathrm{~mL} / \mathrm{min}, \mathrm{p}<0.05$ ) on admission than survivors. Concentrations of high-sensitivity troponin I were 6.9 times $(p<0.0001)$, lactate dehydrogenase 1.4 times $(p<0.01)$, and creatine kinase 4 times $(p<0.0001)$ higher in patients who died than in those who recovered (supplementary material S2).

\section{Outcomes}

Median follow-up time from admission to either death or 21 April 2020 was 26 days (IQR 17.8-31). 34 (31\%) patients died in hospital (Fig 1C): 25 died of COVID-19 pneumonia, 3 died of multiorgan failure secondary to COVID-19 pneumonia, and 6 were palliated at the study end-point on 21 April.

Patients who died had a higher median NEWS2 score on admission (6, IQR 2.5-7.5) than those who recovered (2, IQR 2-6; $p<0.05$ ). Mortality for patients over 65 was $43 \%$ (28) (Table 2). All patients aged under 40 were discharged home. $59 \%$ of patients who died were male (supplementary material S1).

Patients who died were significantly older than those who recovered (mean difference $13.76 \pm 3.12$ years). Several frailty markers were prognostic indicators for death (Table 1). Median CFS was higher in patients over 65 who died than in survivors (5, IQR 4-6 vs 3.5, IQR 2-5; $<<0.01$ ). There was no difference in mortality between sex or BMI category. No patients with underlying heart failure survived the admission $(p<0.0001)$. Current or previous cigarette smoking was predictive of death $(p<0.05)$. The median time between symptom onset and admission had no impact on survival (7 days, IQR 2.3-7.8 vs 4 days, IQR 2-8.5). Admission chest radiograph findings suggestive of COVID-19 infection were not prognostic for death ( $p>0.05$ ).

Ten (59\%) patients aged over 65 who received CPAP survived. All patients aged over 65 years who were deemed to be for full escalation by their treating clinician survived, except one patient who died in intensive care (Table 2).

The majority of patients $(94 ; 87 \%)$ required some form of oxygen therapy, ranging from delivery by nasal cannula to invasive ventilation (Table 3). All 14 (13\%) patients who did not require oxygen were discharged, while $20(61 \%)$ of those requiring more

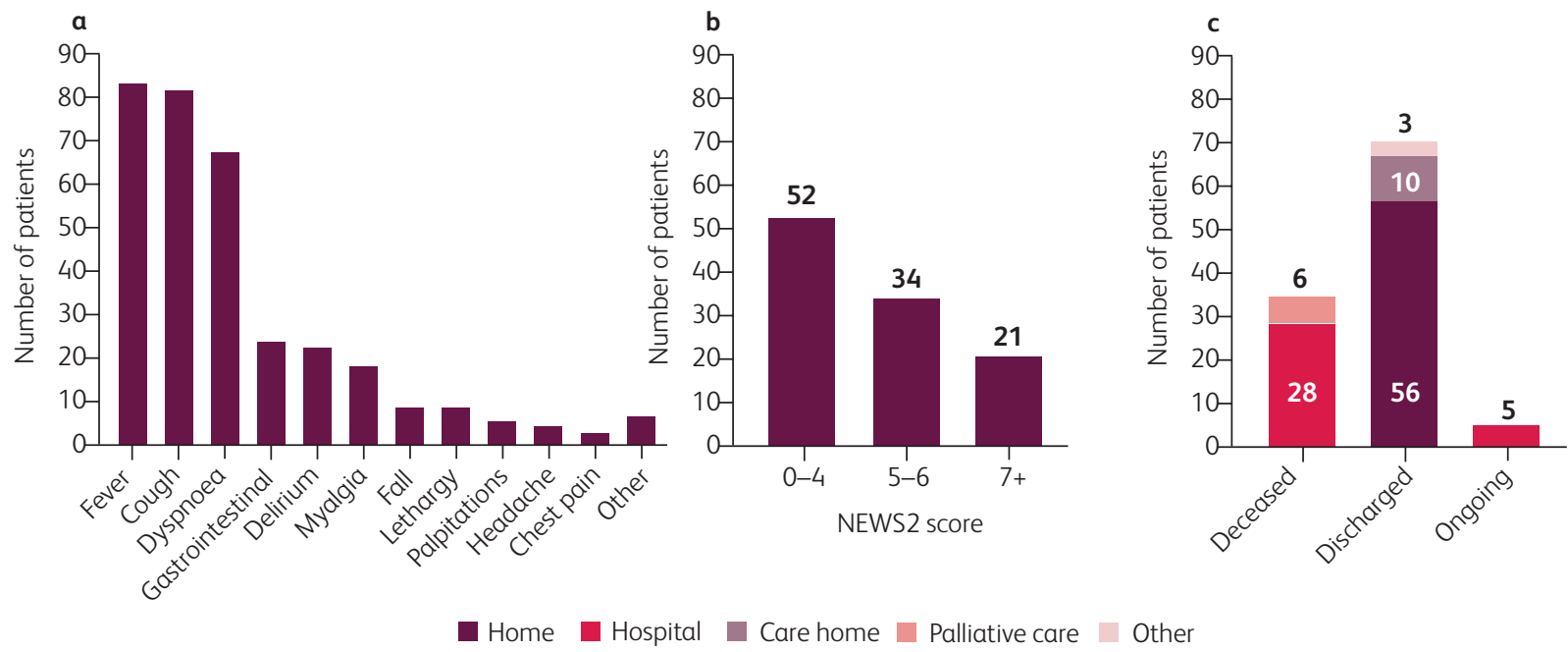

Fig 1. a) Most common presenting complaints. b) NEWS2 score on admission. c) Outcomes. 
Table 2. Escalation status (as decided by treating clinician), and maximum level of care received in deceased and recovered patients in those aged over 65 years. Values are numbers (percentages)

Total $(n=65)$

$17 / 63(27)$

$46 / 63(73)$

$47(72)$

$17(26)$

$1(2)$
Survivors $(n=36)$

Deaths $(n=28)$

Escalation status, age $>65$ years

Full escalation

$15 / 35(43)$

$1 / 27(4)$

DNACPR

Maximum level of care received, age $>65$ years

Ward-based (no CPAP or intubation)

CPAP

Intubation

20/35 (57)

$26 / 27(96)$

One patient over the age of 65 was intubated and remained an inpatient at the study endpoint. CPAP = continuous positive airway pressure; DNACPR $=$ do not attempt cardiopulmonary resuscitation.

than $4 \mathrm{~L} / \mathrm{min}$ died. 19 (73\%) patients who received CPAP therapy recovered and were discharged. The median duration of CPAP was 3 days (IQR 2-7.3). The maximum $\mathrm{FiO}_{2}$ required during CPAP had a median of $60 \%$ (IQR 60-90\%).

In total, nine patients were intubated and required mechanical ventilation. Two were successfully extubated but remained in hospital at the end of the study period, three remained on mechanical ventilation and four had died. The maximum $\mathrm{FiO}_{2}$ required during intubation and ventilation had a median of $80 \%$ (IQR 60-100\%).

Four members of hospital staff were admitted with COVID-19 in March, while 276 of the 699 tested were positive by 21 April.

\section{Follow-up}

Of the 56 patients discharged home, all were alive at the study endpoint. Three patients were readmitted and discharged within the study period: one self-discharged against medical advice; two were discharged without oxygen requirement. All three were readmitted with increasing oxygen requirements and were subsequently discharged.

The social care needs of our cohort increased after COVID-19 admission. Two patients required new packages of care (POC), five required new care home placements, and eight required an increase in mobility aids.
All patients who died after discharge $(4 ; 6 \%)$ were care home residents. Compared with those discharged home, patients discharged to care homes were significantly more likely to die $(p<0.0005)$. Five patients were inpatients at the end of the follow-up period.

\section{Discussion}

This study represents one of the earliest comprehensive analyses of characteristics and outcomes for patients hospitalised with COVID-19 in the UK. It provides vital information applicable to our local population that, to date, has not been defined. The first plausible community transmission within the UK occurred in Haslemere, Surrey, within the catchment area of our hospital. ${ }^{5}$ This has placed us in an ideal position to gather data from the first stages of the outbreak in the UK, capturing our initial efforts to manage this novel disease, and allowing us to adapt early to the rapidly changing healthcare needs of the local population.

With a median age of 71 years, our cohort is older than those studied in China (median 56.0 years), ${ }^{1}$ Italy (median 63.0 years) ${ }^{2}$ and the USA (median 63.0 years). ${ }^{3}$ Age was a significant predictor of mortality: every patient aged under 40 made a full recovery, whereas $43 \%$ of those aged over 65 died (accounting for $85 \%$ of all deaths). With almost 12 million of the UK population over the age of $65,{ }^{10}$ this makes COVID-19 an extreme threat to a large

\begin{tabular}{|c|c|c|c|}
\hline & Total $(n=108)$ & Survivors $(n=69)$ & Deaths $(n=34)$ \\
\hline Median length of stay, days (IQR) & $8(4-13)$ & $8(3-12.5)$ & $9.5(5-16)$ \\
\hline No oxygen & $14(13)$ & $14(20)$ & $0(0)$ \\
\hline Oxygen $\leq 4 \mathrm{~L} / \mathrm{min}$ & $26(24)$ & $23(33)$ & $3(9)$ \\
\hline Oxygen $>4 \mathrm{~L} / \mathrm{min}$ & $33(31)$ & $13(19)$ & $20(59)$ \\
\hline CPAP & $26(24)$ & $19(28)$ & $7(21)$ \\
\hline Median duration of CPAP, days (IQR) & $3(2-7.3)$ & $2(2-6)$ & $6(2-9)$ \\
\hline Invasive ventilation & $9(8)$ & $0(0)$ & $4(12)$ \\
\hline Median duration of invasive ventilation, days (IQR) & $14(7-21)$ & $0(0)$ & $11.5(6-20)$ \\
\hline
\end{tabular}

Five patients remained inpatients at the study endpoint and are not included in survivors or deaths. CPAP = continuous positive airway pressure. 
proportion of the population. This likely explains an in-hospital mortality for patients admitted to hospital of 31\% compared nationally to $32 \%{ }^{4}$ and internationally to $21 \%, 26 \%$, and $28 \%$ in New York, Lombardy and Wuhan respectively. The high in-hospital mortality could be due to higher clinical threshold for admission compared with other countries.

The proportion of patients from non-white ethnicities is lower in Guildford and Waverley (7.2\%) than in England (14.6\%). ${ }^{11}$ This is reflected in this study, where $7 \%$ of patients were black, Asian or from a minority ethnic group. Socioeconomic status was also higher in the study population, with just $0.9 \%$ of local residents claiming unemployment benefits compared with the England rate of $2.8 \% .^{12}$ This is likely to have had an impact on prevalence and transmission of COVID-19 compared with the England population.

Our results are similar to other studies with $59 \%$ of patients who died being male and $41 \%$ female. ${ }^{4}$ After correction for the different admission rates between men and women, the risk of death was not gender dependent. The observed variations could be multifactorial and likely reflect regional differences including age, comorbidities and local demography.

Frailty is widely recognised as a risk factor for poor outcome in patients admitted to hospital. ${ }^{7}$ To the best of our knowledge, this study is the first to analyse the impact of frailty in COVID-19 patients. The majority of frailty markers analysed were prognostic indicators for death, including the presence of a package of care, mobility aids, cognitive impairment, recurrent falls and polypharmacy. CFS was higher in patients who died than in survivors. We also show an increase in social care needs after COVID-19 admission.

Risk factors for severe COVID-19 including chronic obstructive lung disease, coronary heart disease, diabetes and hypertension were initially identified in Wuhan, China. ${ }^{1}$ In our cohort, with the exception of hypertension, these findings were replicated. This study also reports the adverse impact of COVID-19 in those with pre-existing heart failure, or with previous cancer or stroke. The complex interplay between angiotensin-converting enzyme 2 (ACE2), the renin-angiotensin-aldosterone system (RAAS) and COVID-19 may provide some explanation as to why those with cardiovascular disease display such poor outcomes, but mechanisms remain theoretical. ${ }^{13}$

Consistent with international data, ${ }^{1-3}$ lymphopenia was common in those admitted to hospital with COVID-19. The Wuhan study identified raised LDH, troponin, CK, d-dimer and ferritin as prognostic markers for in-hospital mortality.' Our centre formulated a COVID-19 blood panel based on these initial data. We corroborate previous findings that CK, LDH and troponin I levels are significantly higher in non-survivors. We did not replicate the prognostic value of ferritin or d-dimer, although the median values remained above the upper limit of normal on admission. This may be indicative of an inflammatory response and/or cytokine storm. ${ }^{14}$ The elevation of these markers is well documented in sepsis, but their value in COVID-19 remains unclear.

Patients were followed up to either 21 April, or death, for a median of 26 days. To our knowledge, this is the first study reporting outcomes following discharge from hospital including recovery, readmission rate and death in the community.

We have also reported levels of healthcare worker infection and hospitalisation. In line with government guidelines, staff members were tested from 2 April onwards. ${ }^{15}$ In total, 276 of 699 tested healthcare workers were identified as positive on or before $21 \mathrm{April}$. A key aspect of pandemic response is identifying the healthcare worker infection rate in order to guide infection control strategies to maintain a healthy workforce.

Finally, our centre employed a strategy using CPAP to support patients in respiratory failure rather than early intubation and ventilation. With $70 \%$ of patients on CPAP surviving, initial outcome data are promising, although long-term outcomes remain to be determined.

Our study has some limitations. The study population is from a single catchment area (Guildford and Waverley) and, although a complete and comprehensive dataset, it is a smaller cohort size than those in some other studies..$^{2,3}$ While this observational study helps to define the clinical characteristics of patients with COVID-19 in the UK, randomised controlled trials are warranted to assess the efficacy of medical intervention. In the next phase of research, the follow-up of this cohort will help to define the longterm sequelae of COVID-19 infection.

\section{Conclusion}

It remains challenging to determine the prognosis of patients presenting to hospital with early COVID-19. However, our data provide further evidence for the utility of presenting clinical characteristics in clinician judgement. Patients with frailty are particularly vulnerable to COVID-19, reinforcing government policy to protect this group from viral exposure at all costs.

\section{Supplementary material}

Additional supplementary material may be found in the online version of this article at www.rcpjournals.org/clinmedicine: S1 - Presenting characteristics of patients with COVID-19 who died or recovered.

S2 - Admission laboratory parameters of deceased and recovered patients.

\section{Acknowledgements}

We would like to express our sincere gratitude to the patient advisory group for their helpful comments when generating this manuscript. In particular, thank you to the Post-Graduate Medical Education Centre staff for providing us with library space, and to all the hospital staff assisting with data collection. Thank you also to KLH, graduate in mathematics, Massachusetts Institute of Technology, for advice on statistical analysis.

\section{References}

1 Zhou F, Yu T, Du R et al. Clinical course and risk factors for mortality of adult inpatients with COVID-19 in Wuhan, China: a retrospective cohort study. Lancet 2020;395;1054-62.

2 Grasselli G, Zangrillo A, Zanella A et al. Baseline characteristics and outcomes of 1591 patients infected with SARS-CoV-2 admitted to ICUs of the Lombardy region, Italy. JAMA 2020;323:1574-81.

3 Richardson S, Hirsch JS, Narasimhan M et al. Presenting characteristics, comorbidities, and outcomes among 5700 patients hospitalized with COVID-19 in the New York City area. JAMA 2020;323:2052-9.

4 Public Health England. COVID-19: review of disparities in risks and outcomes. PHE: June 2020. www.gov.uk/government/publications/ covid-19-review-of-disparities-in-risks-and-outcomes [Accessed 1 July 2020].

5 BBC News. Coronavirus: latest patient was first to be infected in UK. BBC News, February 2020. www.bbc.co.uk/news/uk-51683428 [Accessed 1 July 2020]. 
6 NHS. Royal Surrey County Hospital: overview. www.nhs.uk/services/ hospitals/overview/defaultview.aspx?id=775 [Accessed 1 July 2020]

7 Hanlon P, Nicholl BI, Jani BD, Lee D, McQueenie R, Mair FS. Frailty and pre-frailty in middle-aged and older adults and its association with multimorbidity and mortality: a prospective analysis of 493 737 UK Biobank participants. Lancet Public Health 2018;3:e323-32.

8 Rockwood K, Song X, MacKnight C et al. A global clinical measure of fitness and frailty in elderly people. CMAJ 2005;173:489-95.

9 British Institute of Radiology. COVID-19 CXR advice sheets. www. bir.org.uk/useful-information/covid-19-resources/covid-19-cxradvice-sheets.aspx [Accessed 1 July 2020]

10 Office for National Statistics. Overview of the UK population: July 2017. ONS: July 2017. www.ons.gov.uk/peoplepopulationandcom munity/populationandmigration/populationestimates/articles/ overviewoftheukpopulation/july2017 [Accessed 1 July 2020].

11 Surrey-i. Guildford and Waverley place-based profile. Surrey County Council, October 2019. www.surreyi.gov.uk/health-profiles/ guildford-and-waverley/ [Accessed 1 July 2020].
12 Surrey-i. Economic indicators for Surrey. Surrey County Council, December 2019. www.surreyi.gov.uk/2019/12/02/economicindicators-for-surrey/ [Accessed 1 July 2020].

13 South AM, Diz DI, Chappell MC. COVID-19, ACE2, and the cardiovascular consequences. Am J Physiol Heart Circ Physiol 2020;318: H1084-90.

14 Chen T, Wu D, Chen $\mathrm{H}$ et al. Clinical characteristics of 113 deceased patients with coronavirus disease 2019: retrospective study. BMJ 2020;368:m1091.

15 Department of Health and Social Care. Coronavirus (COVID-19): getting tested. DHSC, April 2020. www.gov.uk/guidance/coronaviruscovid-19-getting-tested [Accessed 1 July 2020].

Address for correspondence: Dr Aleksandra Kotwica, Royal Surrey County Hospital, Royal Surrey NHS Foundation Trust, Egerton Road, Guildford GU2 7XX, UK.

Email: a.kotwica@doctors.org.uk 